\title{
Modelling and Simulation of Fault detection in Shielded Twisted Pair cables
}

\author{
Gholamhossein Shirkoohi \\ School of Engineering \\ London South Bank University \\ London SE1 0AA, UK \\ Email: maziar.shirkoohi@1sbu.ac.uk
}

\begin{abstract}
Electrical wiring and cables in many suffer damage from time to time. In many cases the damage only affects wire insulation or the shield section of the cables and cannot be detected under normal operating conditions and maintenance inspection. This could in many cases lead to dangerous incidents. This paper discusses computer models of electrical wires, and in particular Shielded Twisted Pair cables, which can be used for developing systems and test procedures based on an Enhanced Time Domain Reflectometry technique, reported earlier, that could lead to identification and inspection for of this type of fault.
\end{abstract}

Keywords - Shielded twisted cable; electrical wiring; insulation and shield faults; modelling of wires

\section{INTRODUCTION}

Time Domain Reflectometry (TDR) technique has, for many years been used to find faults in electrical systems. Faults in electrical wires can arise in many environments, and for different reasons. These environments include residential and corporate structures, road vehicles, surface and underground trains, ships, power plants and mines. However, perhaps by far the most important of these are in aircraft. However, usually the fault detection techniques using TDR would be limited to shorts or open circuit conditions, and not cracks in insulation or minor damage to shields. The technique being developed aims to develop systems that could provide means of detection of small defects in insulation and shielding of cables. The method involves injection of a high frequency pulses into the wires, and the analysis of the subsequent returning reflections.

Although TDR is well known, and is frequently used in many fields, ranging from ecology to detection of faults in microelectronic circuits, the systems developed so far are incapable of detecting minor faults resulting from insulation damage and partial degradation of electrical wires. In aircraft, insulation damage may be caused, for example, by overtightened fasteners and clips holding aircraft wire harnesses in place against the airframe, or due to the rubbing of harnesses against sharp metal edges. On the other hand, cable degradation frequently occurs in aircraft as a result of exposure of the wires to moisture, hydraulic fluids or temperature cycling. These types of fault in wiring harnesses are not detectable by standard TDR techniques. Other techniques have been investigated for finding faults in aircraft harnesses [1-3], communication lines [4], power cables [5] and embedded harness monitoring in aircraft $[6,7]$. These mostly involve considerable change in localised impedance. The detection of small impedance irregularities associated with chafes and frays still remaining elusive [8].

With the soaring increase in computing power in terms of speed of processing operations, storage and random access memory capabilities, and the further advances made in development of modelling software, it has become much easier to predict solutions for engineering problems by carrying out more accurate and appropriate computer simulations with a good degree of precision, and in very little time. Even though, some modelling structures are still difficult to achieve for a realistic representation of what would be considered as simple structures. This paper reports on detailed simulations of a pair of insulated twisted wires, of several metres in length, and a short section of shielded twisted pair version of the wires, using the modern electromagnetic modelling tool, Concerto [9], which was initially developed for $\mathrm{rf}$ and microwave applications [10]. The physical properties of the modelled wires are described in the next section, followed by details of construction and simulation of the models, and discussion of the results obtained.

\section{WIRES INVESTIGATED}

The wires used in this simulation represent a low current electrical twisted pair cable constructed from a pair of parallel wires, forming the so called figure of eight configuration. In this type of twin cable the transmission line capacitance and inductance, and hence the impedance, should remains constant throughout the length of the cable. Hence, any significant change in the localised impedance can be attributed to the fault, which could be anything from an open circuit to a small damage to the insulation. The model for this twin conductor set of wires was carefully twisted using the morphing feature of the modelling facility in concerto in order to obtain a realistic representation of the twisted pair cable. Fig. 1 shows The twisted pair model constructed from two straight wires 


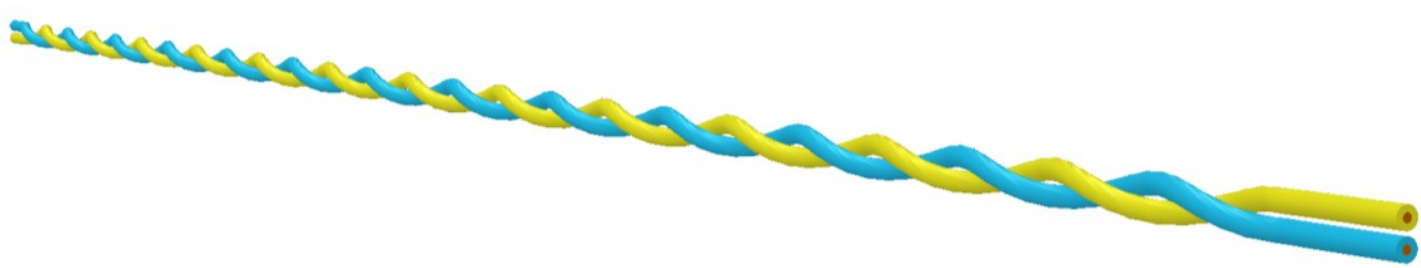

Fig. 1. The twisted pair model constructed from two straight wires forming the core of the Shielded Twisted Pair cable.

forming the core of the Shielded Twisted Pair cable. The conductors are constructed from copper wire with a nominal diameter or $1.00 \mathrm{~mm}$ and an insulation thickness of $0.77 \mathrm{~mm}$.

Several defects were simulated by removing small sections of the insulation on one of the straight wires (without the twist). Initially a large section, and then a smaller $1 \mathrm{~cm}$ section of the insulation were removed from a long (10 metres in length) samples of this type of cables. Although these defects were rather large in comparison with the types of flaw which would be of interest in practise, the study of their effects is of importance prior to those of small cracks and minute insulation damage which are of more significant interest. One of the other important factors to consider is that since the length of the actual cables to be investigated is in excess of twenty or thirty metres, the low energy TDR pulse frequencies need to be limited to around $300 \mathrm{MHz}$. This means that the wavelengths of the pulses are generally much larger than the size of the faults and would not initially give rise to characterisation of the flaws.

\section{MODELLING OF THE TWISTED PAIR WIRES}

The 3D model used to represent the twisted pair cable was the twisted version of the twin cable modelled earlier [11]. In order to achieve this, the straight line figure of eight cable was twisted along its length with a precise twist node periodicity of an actual twisted pair cable of similar cross sectional dimensions.

As previously mentioned, this twisted pair model (Fig. 1) was created from the model of the parallel wires, using the morphing facility in the 3D modeller. A box having the properties of a perfect conductor (PEC) was constructed around the wires, which formed the boundary conditions for the problem, in addition to those of the source and the load ports were assigned, where pulses in the form of radiation could enter the model (from input port). The output port in most cases was set to short circuit as termination. A smaller box was also used close to the twisted pair wires, as in the previous model, in order to increase accuracy of the computation near the wires. Fig. 2 presents the overall geometry and the generated mesh for this model. The front and side faces of these two boxes have been removed in order to display the subdivisions of the volume of the wires and the volume space into cells by the software's mesh generator.

Since the wires are twisted a much finer mesh is needed here. For this reason a much smaller $500 \mathrm{~mm}$ length of cable was considered for rapid analysis.

The twisted pair model was some twenty times smaller than the previous models for the straight line twin cable studied earlier. This was because, as mentioned earlier, the full

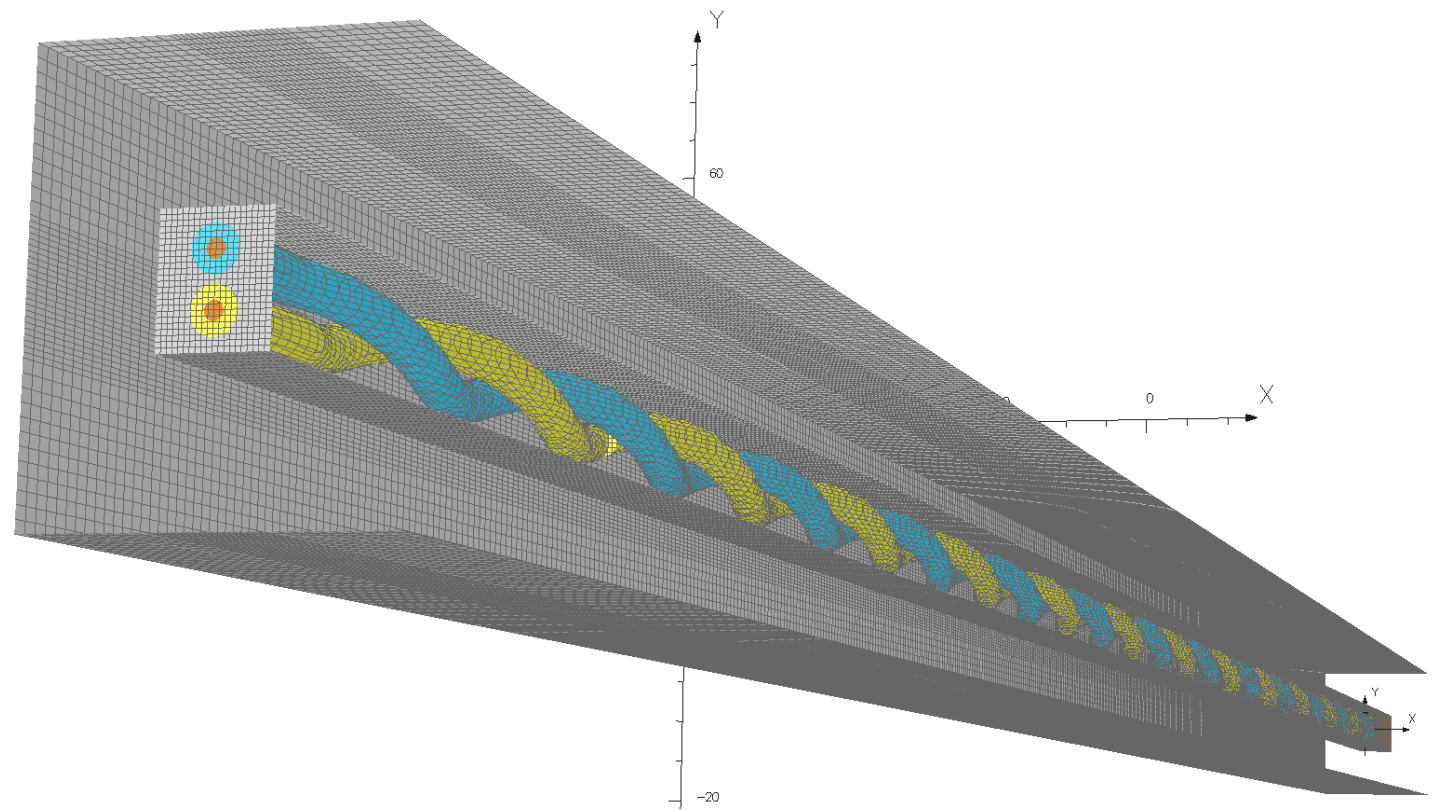

Fig. 2. Model of the $500 \mathrm{~mm}$ twisted pair cable displaying the meshed geometry, the boundary of the solution domain, and the inner, more refined, region for increased accuracy of calculations. 


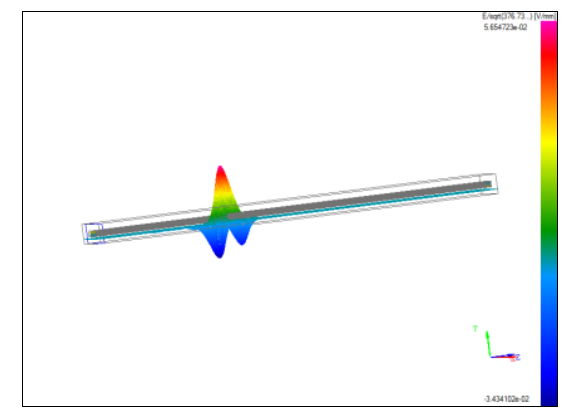

(a)

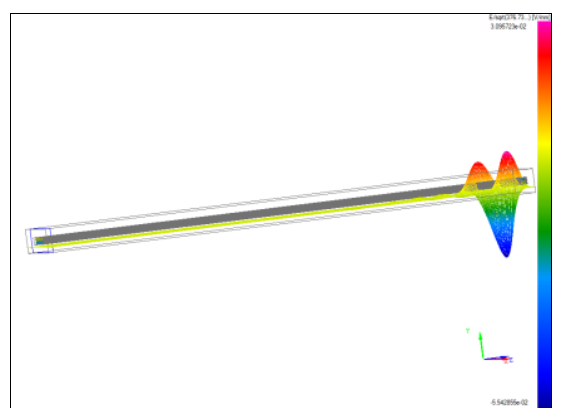

(b)

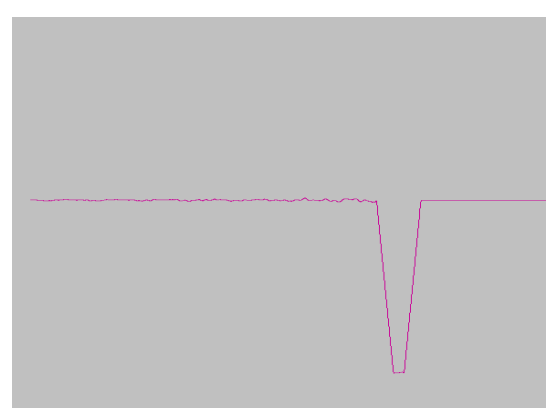

(c)

Fig. 3. Graphical representation of the incident 0.2 ns pulse into the wire pair in QuickWave simulator module of Vector Field's Concerto software; (a) and (b) propagation of the pulse is seen in real-time along the wire, (c) the incident pulse through the cable (as the trace of the vertical component of electric field, $\boldsymbol{E}_{Y}$ )

length model would require a much finer mesh that would demand allocation of far more RAM, CPU time, and would subsequently run progressively slower within the real-time post processor module.

Fig. 3 illustrates graphical representation of the incident 0.2 ns pulse into the twisted wire pair in QuickWave simulator module of Vector Fields Concerto software showing the propagation of the pulse seen in real-time along the wire (a and $\mathrm{b}$ ) and the incident pulse through the cable, as shown in the simulator (c) as the trace of $\boldsymbol{E}_{\boldsymbol{Y}}$, the electric field in y direction and along the cable, representing the incident pulse.

\section{A. Modelling of twisted pair wires with shield and faults}

A great deal of difficulty was encountered in attempting to twist straight conductor pair together with associated shield and insulating sleeve. The shields and sleeve were hence twisted separately with exact twisting pitch as that of the twisted pair model. Fig. 4 presents the construction of the shield and sleeve section. This was then added to the twisted pair model, and combined to form a new unified model. Fig. 5 presents the final assembly of the Shielded Twisted Pair cable from combining the wires and the shield sections.

Initially a $5 \mathrm{~mm}$ section of the shield and sleeve was removed from the model. Fig. 6 displays the simulated cut introduced in the shield and sleeve of the model in order to construct the $5 \mathrm{~mm}$ flaw.

This was then extended to construct a $10 \mathrm{~mm}$ flaw. Fig. 7 shows the cut introduced in the shield and sleeve of the model in order to construct the $10 \mathrm{~mm}$ flaw by further extending the previous fault by an additional 5 millimetres.

\section{B. Simulation of shielded twisted pair wires with fault}

The models were solved using the Quickwave solver module in Vector Fields Concerto software (Cobham).

Once the model was created in the Concerto geometric modeller, it is then submitted to the integrated FDTD solver, known as QuickWave. Fig. 3 illustrates the graphical representation of an incident pulse of 0.2 ns duration, and of a magnitude of around $2 \mathrm{Vpp}$, as it propagates down the cable (shielded twisted pair). The QuickWave software runs in realtime, providing the facility for the propagation of the pulse to be observed while it propagates along the wire; examples are shown in figures 3 ( $a$ and $b$ ), where the pulse is entering the wires from the source port. This pulse is seen to propagate trough the wires and usually dissipated into a matched load. However, for the purpose of the identification of the wire end, the load port boundary condition is usually set to be a short or open circuit, in order to observe an inverted or an upright reflected signal, which indicates the end of the wire. Fig. 3 (c) demonstrates different representation; here, the display presents a selected component, at different positions in space, vs. time. In this case, the component is $\boldsymbol{E}_{\boldsymbol{Y}}$, recorded close to the input end of the cable. When running in TDR mode, the results can be displayed as the raw component, or can be processed in real time to show the impedance change and reflection coefficient vs the time of flight along the line.

For the purpose of examining the reliability of the simulations for practical measurement potentials possibilities, sharper pulses with much higher rise and fall times to those

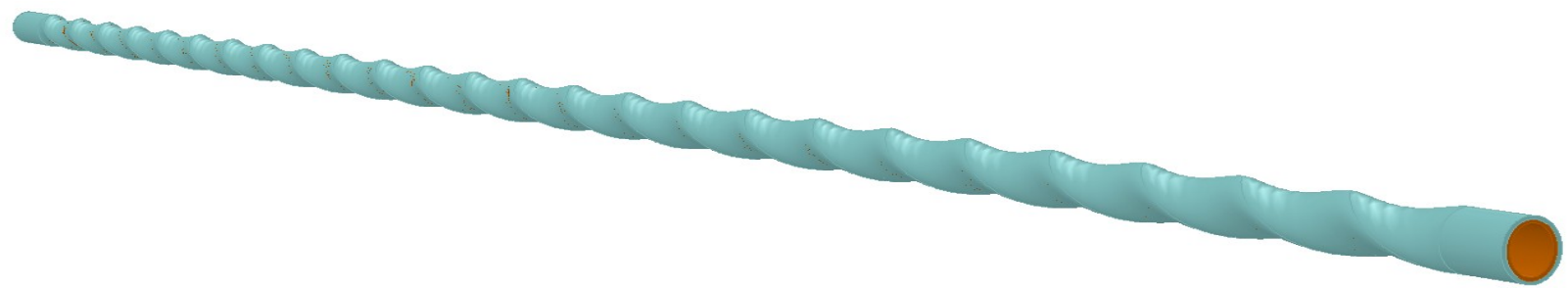

Fig. 4. Model of the shield and sleeve of the cable, separately twisted to exactly fit the twisted inner wires, to form the Shielded Twisted Pair cable. 


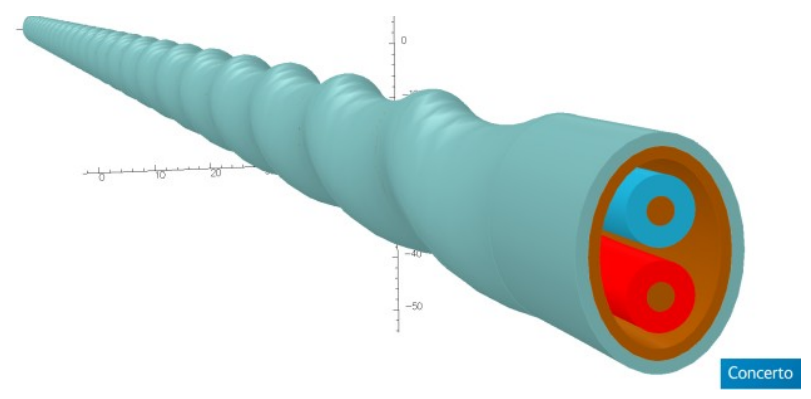

Fig. 5. The final assembly of the shielded Twisted Pair cable from combining the wires and the shield sections.

previously used in measurements were used here. Fig. 8 shows a $0.2 \mathrm{~ns}$ pulse with much higher rise and fall times compared to the one displayed in Fig.3. This pulse shows slight ringing during the rise and fall transitions, as well as clearly visible large ringing in the reflected signal shown on the left of the figure. This normally causes adverse effects in the analysis of the signals when a single trace needs to be scrutinised. However in this case, since the overall results depend on accumulation of several incremental measurements (or rather in this case simulations), since the same ringing effect is present in all incremental pulses, the overall effect is averaged out. This is seen to result in an effective noise, which is not dissimilar, and in fact, much less than those observed in measurements. The pulse would normally propagate through the wires and follows the twists. The helical propagation of the pulse through the twisted wire is also seen to produce a periodic ripple, corresponding to in the periodicity of twists in the wire, which also adds to the noise effect. For identification of the wire end, in these simulations, the load port boundary condition was set to be an open circuit, in order to observe an upright reflected signal which indicated the end of the wire.

\section{DISCUSSIONS}

Fig. 9 presents analysis of responses for for only as few as 16 consecutive pulses simulated in Quickwave for a defect of 5

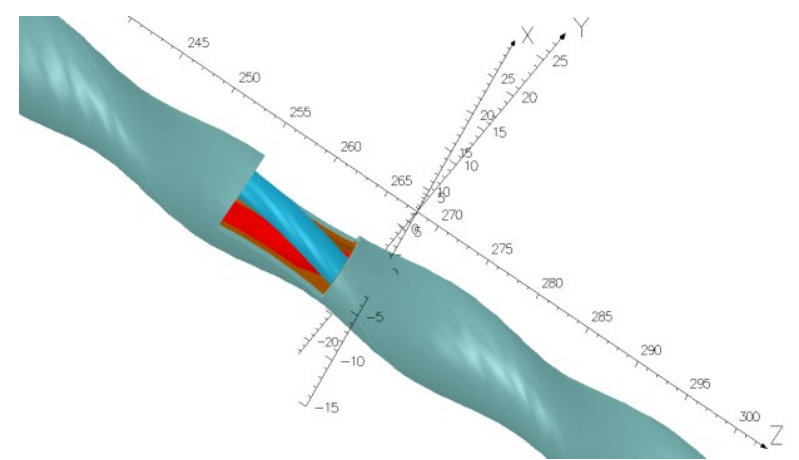

Fig. 7. The $10 \mathrm{~mm}$ cut introduced in the shield and sleeve of the model, by extending the $5 \mathrm{~mm}$ flaw in order to construct the $1 \mathrm{~cm}$ defect.

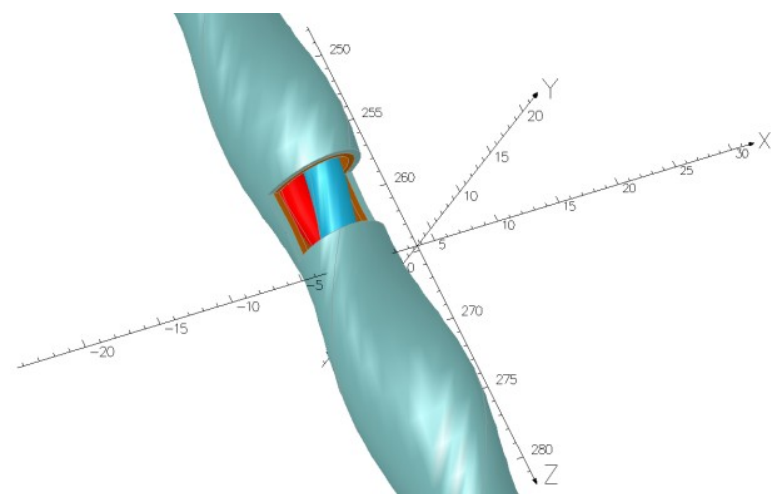

Fig. 6. The cut introduced in the shield and sleeve of the model in order to construct the $5 \mathrm{~mm}$ flaw.

$\mathrm{mm}$ in a location close to centre of a $500 \mathrm{~mm}$ long shielded twisted wire cable. The results in this figure divulge significant change in the cumulated voltage values resulting from small change in the impedance of the cable due to the flaw which increases in value with rising number of incremental pulses used. Some noise is also clearly present in the figure which is expected, however, this is much smaller in magntude as those expected in actual measurements which are usually very high in magnitude, but the signal from the flaw presented here is arund 2 volts in magnitude which is much higher than the noise expected in measurements. The amplitude of this analysed, cumulative inceremental increases with inceasing numbrer of incremental pulses, which could be as many as 200 in number. The distinctive change in the voltage pattern is observed close to the centre of the cable where the flaw was introduced.

Fig. 10 presents analysis of responses for consecutive pulses simulated in Quickwave for a defect of $1 \mathrm{~cm}$ in the sleeve and shield screen of the shielded twisted pair cable at a position close to the centre of the cable. Again similar changes in the analysis of the cumulative results is observed. The results in this figure show a more prominent change in the voltage values than those observed in Fig.8, as expected, since these result from the change in the impedance of the cable due to a larger flaw. In comparison to the results obtained for the

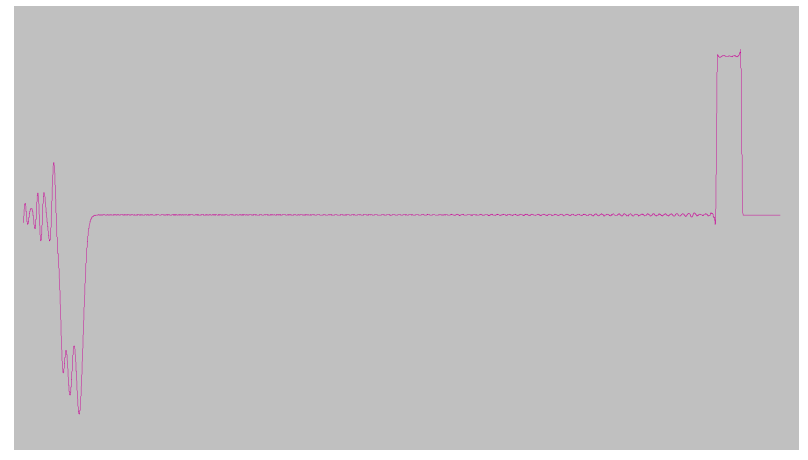

Fig. 8. The 0.2 ns pulse with high rise and fall times used in simulations resulting in ringing close to the pulse edges and in the reflected signal. 


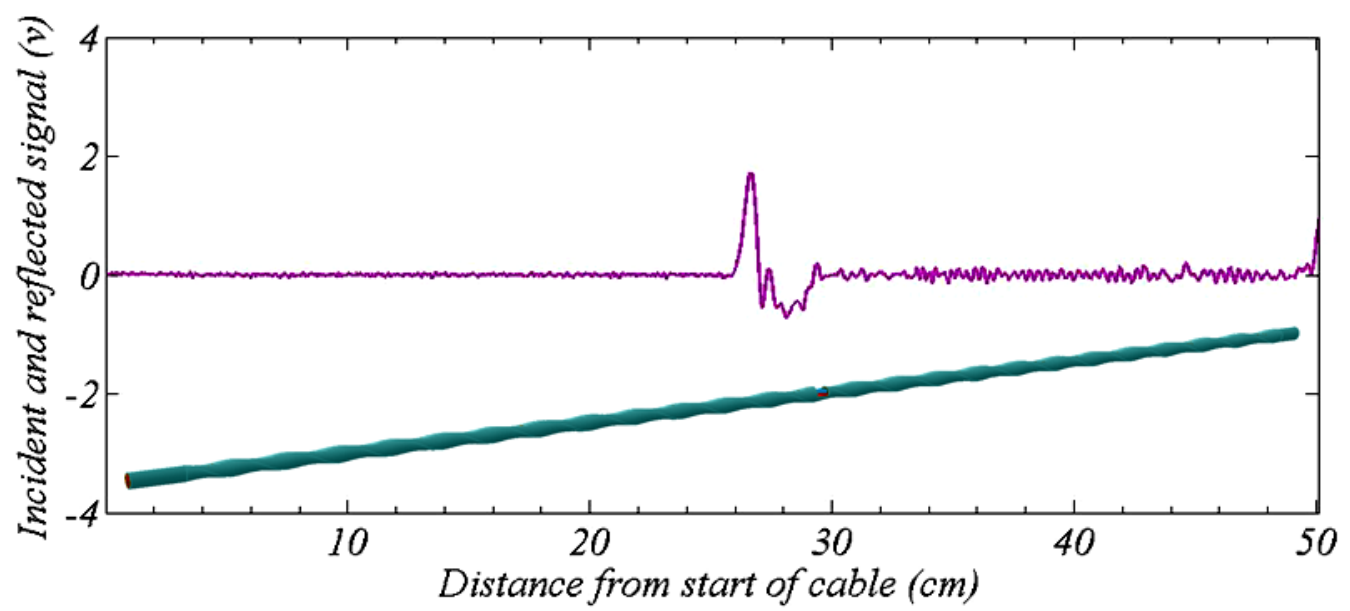

Fig. 9. Analysed response for the Shielded Twisted pair Cable for the simulated fault on sleeve and shield, for a $5 \mathrm{~mm}$ defect on Shield and sleeve.

straight cables, larger change is observed for the the shielded twisted pair cable, since in this case the capacitive component of the impedance is very dominant in shielded cables. The signals also increases in value with rising number of incremental pulses used, not shown here, but follows similar pattern presented previously. It is restated again here that due to the complexity of the shielded twisted pair model, and the necessity for higher resolution for the discretisation in the required high frequency mesh, the model constructed for the shielded twisted pair cable was much smaller than those of cable lengths used for measurements. This was also the case for the models constucted to simulate similar faults, particularly in coaxial cables, presented in [11], where, the confirmation of the modelling results, using the exactly the same measurement condtions in the simulation, as those used in measuremets were confirmed. This was in a direct comparison to those obtained by measurements in similar lengths of cables. This was demonstrated for at least two different types of cable geometric topologies, namely the straight insulated conductor pairs and shielded coaxial cables. Results for the measurements in $5 \mathrm{~mm}$ and $10 \mathrm{~mm}$ faults obtained from the simulations here are also similar to those reported in measurments carried for the coaxial cables [13].

The predicted fault location matches exactly with that of the location identified by the measurement results. The shielded twisted pair cables also suffer from small changes in localised impedances due to the periodic twists in the longitudinal direction, resulting in ripple effect.

\section{CONCLUSIONS}

Models of shielded twisted pair electrical cables with flaws in shield were constructed, and were subjected to simulated TDR tests in the QuickWave FDTD solver. A short length of shielded twisted pair cable was investigated. The outcome of the simulations show that significant variations in the comparative results can be observed, even though the TDR pulse width was much larger than the size of the flaw. The

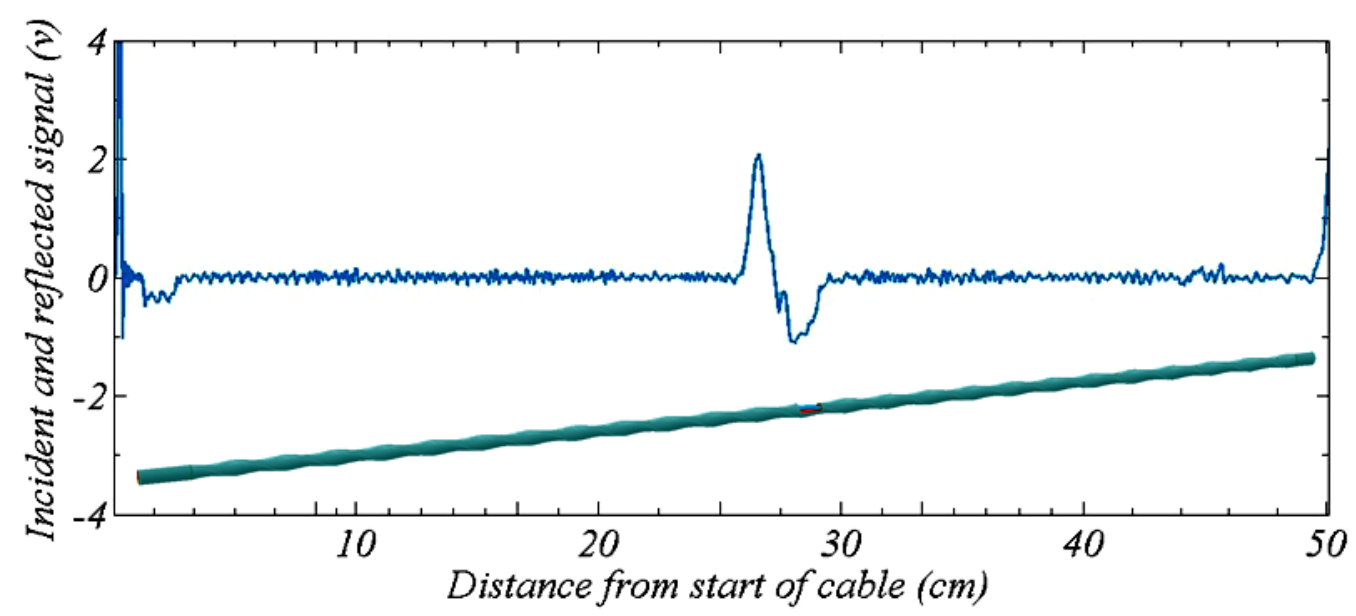

Fig. 10. Analysed response for the Shielded Twisted pair Cable for the simulated fault on sleeve and shield, for a $10 \mathrm{~mm}$ defect on Shield and sleeve only. 
modelling results can be used in order to develop more elaborate experimental techniques based on TDR method in order to detect small faults in wire insulation.

The paper also shows that modelling results obtained can be used in order to develop similar non-standard experimental techniques (in this case based on TDR method), in order to detect small faults in wire insulation. This technique can be used to predict defects in wires and cables.

\section{FUTURE WORK}

Extensive work on analysis of results obtained based on both modelling and measurements are in progress in order to fully validate the nature of the incremental pulse technique for detection of small faults in insulation and shielding sections of electrical wiring and shielded cables.

\section{REFERENCES}

[1] Furse, C., Chung, Y.C., Dangol, R., Nielsen, M., Mabey, G., and Woodward, R., "Frequency-Domain Reflectometry for on-Board Testing of Aging Aircraft Wiring," IEEE Transactions on Electromagnetic Compatibility, Vol. 45, No.2, pp. 306-315, May 2003.

[2] Smith, P., Furse, C., and Gunther, J.,"Analysis of Spread Spectrum Time Domain Reflectometry for Wire Fault Location," IEEE Sensors Journal, Vol. 5, No. 6, pp. 1469-1478, December 2005.

[3] Shin, Y.J., Powers, E.J., Choe, T.S., Hong, C.Y., Song, E.S., Yook, J.G., Park, J.B., "Application of Time-Frequency Domain Reflectometry for Detection and Localization of a Fault on a Coaxial Cable," IEEE Transactions on Instrumentation and Measurement, Vol. 54, No. 6, Dec. 2005.
[4] Dodds, D.E., "Single-Ended FDR to Locate and Specifically Identify DSL Loop Impairments", IEEE International Conference on Communications 2007 (ICC'07), pp. 6413 - 6418, June 2007.

[5] Navaneethan, S., Soraghan, J.J., Siew, W.H., McPherson, F., and Gale, P.F. "Automatic fault location for underground low voltage distribution networks," IEEE Trans. Power Del., vol. 16, no. 2, pp. 346-351, Apr. 2001.

[6] Auzanneau, F., "Embedded Aeronautic Harness Monitoring and Reliability," International Conference Advanced Aircraft Cabling, Hamburg, Germany, Jul 2014.

[7] Ben Hassen, W., Auzanneau, F., et al, "Distributed Sensor Fusion for Wire Fault Location Using Sensor Clustering Strategy," International Journal of Distributed Sensor Networks, Vol. 2015, Article ID 538643, pp. 1-17, Jan 2015.

[8] Griffiths, L.A., Parakh, R., Furse, C., and Baker, B.,"The Invisible Fray: A Critical Analysis of the Use of Reflectometry for Fray Location," IEEE Sensors Journal, Vol. 6, No. 3, pp. 697 706, June 2006.

[9] CONCERTO 7 Reference Manual, Vector Fields Ltd, Oxford, 2008.

[10] Suidong Yang, Michaelides, A., Riley, C., Archer, J., Hook, M., Simkin, J, "Optimizing the design examples of wide-band antennas," Wideband and Multi-band Antennas and Arrays, 2005, IEE (Ref. No. 2005/11059), pp. 185-190, Sept. 2005.

[11] Shirkoohi, G., "Modelling of fault detection in electrical wiring," IET Journal of Science, Measurement \& Technology, Vol. 9, No. 2, pp. 211-217, 2015.

[12] Shirkoohi, G., Hasan, K., "Enhanced TDR Technique for Fault Detection in Electrical Wires and Cables," 2nd International Symposium on NDT in Aerospace 2010, tu2b3, Hamburg, Germany, pp. 1-6, Nov 2010.

[13] Shirkoohi, G., "Fault detection in aircraft wiring using enhanced multi-pulse TDR technique"7th International Symposium on NDT in Aerospace 2015, mo5a6, Bremen, Germany, pp. 1-8, Nov. 2015. 